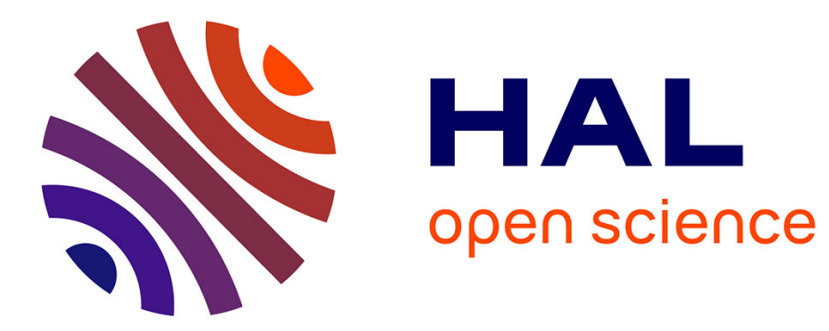

\title{
Complex Multiply-Add and Other Related Operators
}

Milos Ercegovac, Jean-Michel Muller

\section{To cite this version:}

Milos Ercegovac, Jean-Michel Muller. Complex Multiply-Add and Other Related Operators. SPIE Conf. Advanced Signal Processing Algorithms, Architectures and Implementation XVII, 6697, SPIE, Aug 2007, San Diego, United States. ensl-00167372

\section{HAL Id: ensl-00167372}

\section{https://hal-ens-lyon.archives-ouvertes.fr/ensl-00167372}

Submitted on 20 Aug 2007

HAL is a multi-disciplinary open access archive for the deposit and dissemination of scientific research documents, whether they are published or not. The documents may come from teaching and research institutions in France or abroad, or from public or private research centers.
L'archive ouverte pluridisciplinaire HAL, est destinée au dépôt et à la diffusion de documents scientifiques de niveau recherche, publiés ou non, émanant des établissements d'enseignement et de recherche français ou étrangers, des laboratoires publics ou privés. 


\title{
Complex Multiply-Add and Other Related Operators
}

\author{
Miloš D. Ercegovac ${ }^{a}$ and Jean-Michel Muller ${ }^{b}$ \\ ${ }^{a}$ Computer Science Department, UCLA, Los Angeles, CA 90025, USA \\ ${ }^{b}$ ENS-Lyon, France
}

\begin{abstract}
In this work we present algorithms and schemes for computing several common arithmetic expressions defined in the complex domain as hardware-implemented operators. The operators include Complex Multiply-Add $(C M A: a b+c)$, Complex Sum of Products $(C S P: a b+c e+f)$, Complex Sum of Squares $\left(C S S: a^{2}+b^{2}\right)$, and Complex Integer Powers $\left(C I P k: x^{2}, x^{3}, \ldots, x^{k}\right)$. The proposed approach is to map the expression to a system of linear equations, apply a complex-to-real transform, and compute the solutions to the linear system using a digit-by-digit, the most significant digit first, recurrence method. The components of the solution vector corresponds to the expressions being evaluated. The number of digit cycles is about $m$ for $m$-digit precision. The basic modules are similar to left-to-right multipliers. The interconnections between the modules are digit-wide.
\end{abstract}

Keywords: Complex arithmetic, multiply-add, sum of products, sum of squares, integer powers

\section{INTRODUCTION}

With the exception of complex addition and multiplication, $, ?, ?$ complex online SVD, ${ }^{?}$ complex operations are typically not implemented in hardware. Recently, hardware-oriented methods for complex division and square root have been introduced.?,?

In this paper we describe a new method for computing several common arithmetic expressions defined in the complex domain, suitable for hardware implementation as operators. The operators include Complex MultiplyAdd $(C M A: a b+c))$, Complex Sum of Products $(C S P: a b+c e+f)$, Complex Sum of Squares $(C S S$ : $\left.a^{2}+b^{2}\right)$, and Complex Integer Powers $\left(C I P k: x^{2}, x^{3}, \ldots, x^{k}\right)$. The variables and results are fixed-point complex numbers. The proposed approach is to map the expression to a system of linear equations, apply a complex-to-real transform, and compute the solutions to the linear system using a digit-by-digit, the most significant digit first method. The components of the solution vector corresponds to the expressions being evaluated. The number of digit cycles is about $m$ for $m$-digit precision. The basic modules are similar in complexity to left-to-right multipliers. The interconnections between the modules are digit-wide. The proposed method is a generalization of a polynomial evaluation method over the reals introduced as the E-method,,$?$ ? and recently discussed in.? This paper is based on the report ${ }^{?}$ where the complex E-method is introduced and discussed in general terms.

The method uses the following approach: (i) an expression is mapped onto a system of linear equations, (iii) a transform is applied to change the complex domain to the real domain, and (iii) the system is solved in a digit-by-digit manner, the most-significant digit first.

We first review the transform which allows the method to be used in the complex field $\mathbf{C}$ as discussed in. Then we show how to use the complex evaluation method (CE-method) in implementing complex operators $C M A, C S P, C S S$, and $C I P$.

Further author information: (Send correspondence to M. Ercegovac, milos@cs.ucla.edu) 


\section{COMPLEX-REAL (CR) TRANSFORMS}

Complex numbers can be represented by $2 \times 2$ skew-symmetric matrices

$$
x+i y \leftrightarrow\left(\begin{array}{cc}
x & -y \\
y & x
\end{array}\right)
$$

This isomorphism holds for complex addition and multiplication which are used in the proposed method.

Consequently, an $m \times n$ matrix of complex numbers can be represented as a $2 m \times 2 n$ matrix of real numbers. For $n \times n$ complex matrices, considered in this paper, the transform from the complex domain to the real domain is as follows.

The CR-transform of a $n$-dimensional complex linear system is a $2 n$-dimensional real linear system. For example, let $n=$, then the CR transform is

$$
\begin{array}{r}
\left(\begin{array}{lll}
a_{1,1} & a_{1,2} & a_{1,3} \\
a_{2,1} & a_{2,2} & a_{2,3} \\
a_{3,1} & a_{3,2} & a_{3,3}
\end{array}\right) \times\left(\begin{array}{c}
s_{1} \\
s_{2} \\
s_{3}
\end{array}\right)=\left(\begin{array}{c}
t_{1} \\
t_{2} \\
t_{3}
\end{array}\right) \\
\left(\begin{array}{cccccc}
a_{1,1}^{r} & -a_{1,1}^{i} & a_{1,2}^{r} & -a_{1,2}^{i} & a_{1,3}^{r} & -a_{1,3}^{i} \\
a_{1,1}^{i} & a_{1,1}^{r} & a_{1,2}^{i} & a_{1,2}^{r} & a_{1,3}^{i} & a_{1,3}^{r} \\
a_{2,1}^{r} & -a_{2,1}^{i} & a_{2,2}^{r} & -a_{2,2}^{i} & a_{2,3}^{r} & -a_{2,3}^{i} \\
a_{2,1}^{i} & a_{2,1}^{r} & a_{2,2}^{i} & a_{2,2}^{r} & a_{2,3}^{i} & a_{2,3}^{r} \\
a_{3,1}^{r} & -a_{3,1}^{i} & a_{3,2}^{r} & -a_{3,2}^{i} & a_{3,3}^{r} & -a_{3,3}^{i} \\
a_{3,1}^{r} & a_{3,1}^{i} & a_{3,2}^{r} & a_{3,2}^{i} & a_{3,3}^{r} & a_{3,3}^{i}
\end{array}\right) \times\left(\begin{array}{c}
s_{1}^{r} \\
s_{1}^{i} \\
s_{2}^{r} \\
s_{2}^{i} \\
s_{3}^{r} \\
s_{3}^{i}
\end{array}\right)=\left(\begin{array}{c}
t_{1}^{r} \\
t_{1}^{i} \\
t_{2}^{r} \\
t_{2}^{i} \\
t_{3}^{r} \\
t_{3}^{i}
\end{array}\right)
\end{array}
$$

where $a_{j, k}=a_{j, k}^{r}+i a_{j, k}^{i}, s_{j}=s_{j}^{r}+i s_{j}^{i}$ and $t_{j}=t_{j}^{r}+i t_{j}^{i}$. These two linear systems are equivalent.

The real linear system (??) is obtained from the complex linear system (??) by replacing each complex element by the $2 \times 2$ matrix defined in (??). In the following sections we review a hardware-oriented method for solving such a system.

\section{REAL E-METHOD}

For simplicity, we discuss here radix-2 E-method. Adaptation to higher radices is straightforward. The radix-2 method consists in solving the $n$-dimensional linear system

$$
A s=v
$$

using the following iteration on residuals:

$$
w^{(j)}=2 \times\left[w^{(j-1)}-A d^{(j-1)}\right]
$$

with $w^{(0)}=\left[v_{0}, v_{1}, \ldots, v_{n}\right]^{T}$, and $d^{(j)}=\left[d_{0}, d_{1}, \ldots, d_{n}\right]^{T}$ where the digits $d_{k}^{(j)}$ are in $\{-1,0,1\}$. Define the number $D_{k}^{(j)}=d_{k}^{(0)} \cdot d_{k}^{(1)} d_{k}^{(2)} \ldots d_{k}^{(j)}$ (the $d_{k}^{(j)}$ are the digits of a radix-2 signed-digit representation of $D_{k}^{(j)}$ ). By induction,

$$
w^{(j)}=2^{j}\left[w^{(0)}-A D^{(j-1)}\right] .
$$

Using (??), one can show that if the residuals $\left|w_{k}^{(j)}\right|$ are bounded, then for all $k, D_{k}^{(j)}$ converges to $s_{k}$ as $j$ goes to infinity. At step $j$ we must select a value of the digits $d_{k}^{(j)}$ from the residuals $w_{k}^{(j)}$ such that the values $w_{k}^{(j+1)}$ remain bounded. The following selection function, proposed in ${ }^{?}$ as a form of rounding, achieves such a choice.

$$
S E L(x)=\left\{\begin{array}{lr}
\operatorname{sign} x \times\lfloor|x+1 / 2|\rfloor, & \text { if }|x| \leq 1 \\
\operatorname{sign} x \times\lfloor|x|\rfloor, & \text { otherwise, }
\end{array}\right.
$$


To avoid carry-propagate addition in the recurrence, the selection function is applied to an estimate of the residual : $d_{k}^{(j)}=S E L\left(\hat{w}_{k}^{(j)}\right)$, where $\hat{w}_{k}^{(j)}$ is a low-precision approximation to $w_{k}^{(j)}$.

The iterations converge to the desired result if residual vector $w^{(j)}$ is bounded. Let $\xi, \alpha$ and $\Delta$ (with $0 \leq \Delta<1$ ) be constants such that

1. Sum of magnitudes of off-diagonal elements in matrix $A$ : $\leq \alpha$;

2. Magnitudes of right-hand side elements: $\leq \xi$;

3. $\left|w_{k, r}^{(j)}-\hat{w}_{k, r}^{(j)}\right| \leq \frac{\Delta}{2}$, and $\left|w_{k, i}^{(j)}-\hat{w}_{k, i}^{(j)}\right| \leq \frac{\Delta}{2}$

Since $\left|d_{k, r}^{(j-1)}-\hat{w}_{k, r}^{(j-1)}\right| \leq 1 / 2$ and $\left|d_{k, i}^{(j-1)}-\hat{w}_{k, i}^{(j-1)}\right| \leq 1 / 2$, from (??) we find

$$
\left|w_{k, r}^{(j)}\right| \leq 2\left(\frac{1}{2}+\frac{\Delta}{2}+\alpha\right)=1+\Delta+2 \alpha .
$$

The same bound holds for $\left|w_{k, i}^{(j)}\right|$. For this bound to be feasible, we must assure that a suitable choice of $d_{k, r}^{(j)}$ and $d_{k, r}^{(j)}$ in $\{-1,0,1\}$ is possible. This requires that $\left|w_{k, r}^{(j)}\right|$ and $\left|w_{k, i}^{(j)}\right|$ should be less than $3 / 2$. Therefore,

$$
\Delta+2 \alpha \leq \frac{1}{2}
$$

Since $\left|w_{k, r}^{(0)}\right|$ and $\left|w_{k, i}^{(0)}\right|$ must also be less than $3 / 2$, we get

$$
\xi \leq \frac{3}{2}
$$

In the complex E-method the coefficient matrix $A$, the solution vector $s$ and the right-hand side $v$ are in the complex domain. Moreover, the complex residual vector is denoted as

$$
w^{(j)}=\left[w_{0, r}^{(j)}, w_{0, i}^{(j)}, w_{1, r}^{(j)}, w_{1, i}^{(j)} \cdots, w_{n, r}^{(j)}, w_{n, i}^{(j)}\right]
$$

and the complex digit vector $d^{(j)}$ is written as

$$
d^{(j)}=\left[d_{0, r}^{(j)}, d_{0, i}^{(j)}, d_{1, r}^{(j)}, d_{1, i}^{(j)}, \cdots, d_{n, r}^{(j)}, d_{n, i}^{(j)}\right]
$$

The mapping of operators on linear systems and complex residual iterations are discussed in the following sections for each complex operator.

\section{COMPLEX MULTIPLY-ADD OPERATOR ( $C M A)$}

The operator computes $y=a b+c$. In the real domain, the mapping to a linear system is

$$
\left(\begin{array}{cc}
1 & -a \\
0 & 1
\end{array}\right) \times\left(\begin{array}{c}
s_{0} \\
s_{1}
\end{array}\right)=\left(\begin{array}{c}
c \\
b
\end{array}\right)
$$

where the solution is obtained as $s_{0}=y$. In the complex domain, the variables are $y=y^{r}+i y^{i}, a=a^{r}+i a^{i}$, $b=b^{r}+i b^{i}$, and $c=c^{r}+i c^{i}$. The mapping in this case is

$$
\left(\begin{array}{cccc}
1 & 0 & -a^{r} & a^{i} \\
0 & 1 & -a^{i} & -a^{r} \\
0 & 0 & 1 & 0 \\
0 & 0 & 0 & 1
\end{array}\right) \times\left(\begin{array}{c}
s_{0}^{r} \\
s_{0}^{i} \\
s_{1}^{r} \\
s_{0}^{i}
\end{array}\right)=\left(\begin{array}{c}
c^{r} \\
c^{i} \\
b^{r} \\
b^{i}
\end{array}\right)
$$

so that $s_{0}^{r}=y^{r}$ and $s_{0}^{i}=y^{i}$. 
The residual recurrences are:

$$
\begin{aligned}
& w_{0, r}^{(j)}=2\left[w_{0, r}^{(j-1)}-d_{0, r}^{(j-1)}+a^{r} d_{1, r}^{(j-1)}-a^{i} d_{1, i}^{(j-1)}\right] \\
& w_{0, i}^{(j)}=2\left[w_{0, i}^{(j-1)}-d_{0, i}^{(j-1)}+a^{i} d_{1, r}^{(j-1)}+a^{r} d_{1, i}^{(j-1)}\right] \\
& w_{1, r}^{(j)}=2\left[w_{1, r}^{(j-1)}-d_{1, r}^{(j-1)}\right] \\
& w_{1, i}^{(j)}=2\left[w_{1, i}^{(j-1)}-d_{1, i}^{(j-1)}\right]
\end{aligned}
$$

with the initial conditions

$$
w_{0, r}^{(0)}=c^{r}, \quad w_{0, i}^{(0)}=c^{i}, \quad w_{1, r}^{(0)}=b^{r}, \quad w_{1, i}^{(j)}=b^{i}
$$

The convergence requires that the following conditions are satisfied

$$
\left|a^{r}\right|+\left|a^{i}\right| \leq \alpha, \quad\left|c^{r}\right|,\left|c^{i}\right|,\left|b^{r}\right|,\left|b^{i}\right| \leq 3 / 2
$$

From condition (??) and using $\Delta=1 / 8$, we get $\alpha \leq 3 / 16$. Therefore, $\left|a^{r}\right|,\left|a^{i}\right| \leq 3 / 32$ assures convergence of the algorithm. This range reduction of the input $a$ can be achieved by scaling of the initial value.?

A scheme for implementing the $C M A$ operator is shown in Figure ??(a) and the corresponding elementary unit (EU0) is illustrated in Figure ??(b). Elementary units $E U 1$ can be simplified as discussed below. A bitparallel bus transmits $a$ values in a broadcast mode, while $b$ and $c$ variables are loaded in separate cycles. Note that the initialization cycles could be shorter than the iteration cycles.
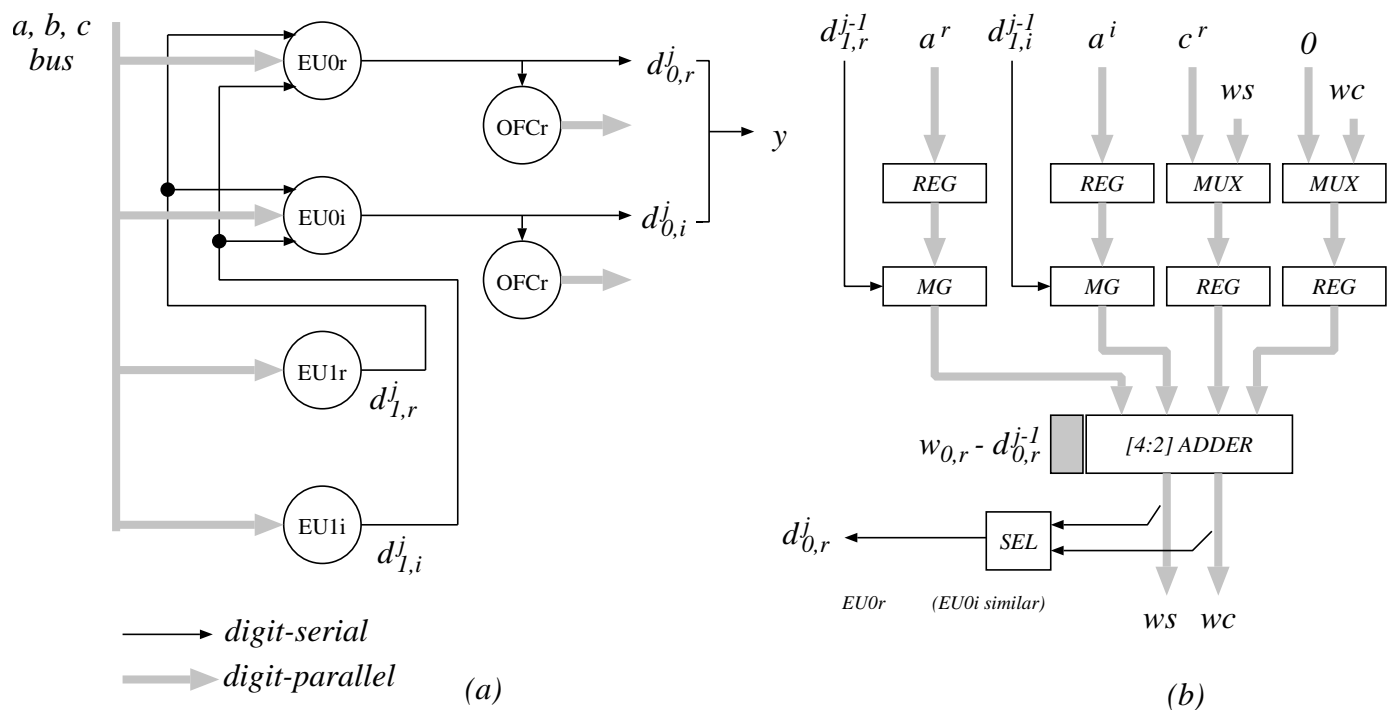

Figure 1. (a) Scheme for $C M A$ operator. (b) Block diagram of elementary unit.

A block diagram of an elementary unit EU0 (real part only) for CMA operator is shown in Figure ??(b). It uses the following modules:

- Registers (4)

- Multiple generators MG (2), producing $\{-1,0,1\} \times a^{r}$ and $\{-1,0,1\} \times a^{i}$, and buffers 
- Multiplexers MUX (2) for initializing the residual

- A [4:2] adder (the shaded MS part performs the indicated subtraction of the selected digit)

- Output digit selection $S E L$ (a small table or a gate network)

The elementary unit EU1 can be greatly simplified. This module, in general radix- $r$ case is effectively a recoder from the digit set $\{0,1, \ldots, r-1\}$ to the the set $\{-a, \ldots, a\}$ where $r / 2 \leq a \leq r-1$. In the case of radix-2, this recoding is unnecessary: the module is a left shift register.

The digit-serial outputs of $E U$ s can be converted into digit-parallel form using on-the-fly converters $O F C r$ and $O F C i$ as indicated by the thick lines?

The cycle time (without interconnect delay between units), in terms of a full adder delay $t$, is estimated as

$$
\begin{aligned}
T_{E U-C M A} & =t_{B U F F}+t_{M G}+t_{S E L}+t_{[4: 2]}+t_{R E G} \\
& \approx(0.4+0.3+1+1.3+0.9) t=3.9 t
\end{aligned}
$$

The cost, again in terms of area of a full adder $A_{F A}$, is estimated as

$$
\begin{aligned}
A_{E U-C M A}(m) & =A_{S E L}+2 A_{B U F F}+(m+2)\left[2 A_{M G}\right. \\
& \left.+2 A_{M U X}+A_{[4: 2]}+4 A_{R E G}+A_{O F C}\right] \\
& \approx[5+2 \times 0.4+(m+2)(4 \times 0.45 \\
& +2.3+4 \times 0.6+2.1)] A_{F A} \\
& \approx(23+9 m) A_{F A}
\end{aligned}
$$

The cost is estimated as area occupied by modules using the area of a full-adder $A_{F A}$ as the unit. The areas of primitive modules are: Register $A_{R E G}=0.6 A_{F A}$; buffer $A_{B U F F}=0.4 A_{F A}$; MUX $A_{M U X}=0.45 A_{F A}$; multiple generator MG $A_{M G}=0.45 A_{F A} ;[4: 2]$ adder $A_{[4: 2]}=2.3 A_{F A}$; SEL $A_{S E L}=5 A_{F A}$, and on-the-fly converters $A_{O F C}=2 A_{M U X}+2 A_{R E G}=2.1 A_{F A}$. A total cost of an $m$-bit $C M A$ operator is

$$
A_{C M A}(m)=2 \times A_{E U-C M A}(m)+2 \times(m+2) A_{R E G} \approx(50+20 m) A_{F A}
$$

\section{COMPLEX SUM OF PRODUCTS OPERATOR (CSP)}

The operator computes $y=a b+c e+f$. In the real domain, the mapping to a linear system is

$$
\left(\begin{array}{ccc}
1 & -a & -c \\
0 & 1 & 0 \\
0 & 0 & 1
\end{array}\right) \times\left(\begin{array}{l}
s_{0} \\
s_{1} \\
s_{2}
\end{array}\right)=\left(\begin{array}{l}
f \\
b \\
e
\end{array}\right)
$$

and the solution $s_{0}=y$. In the complex domain, $a=a^{r}+i a^{i}, b=b^{r}+i b^{i}$, etc. The mapping in this case is

$$
\left(\begin{array}{cccccc}
1 & 0 & -a^{r} & a^{i} & -c^{r} & c^{i} \\
0 & 1 & -a^{i} & -a^{r} & -c^{i} & -c^{r} \\
0 & 0 & 1 & 0 & 0 & 0 \\
0 & 0 & 0 & 1 & 0 & 0 \\
0 & 0 & 0 & 0 & 1 & 0 \\
0 & 0 & 0 & 0 & 0 & 1
\end{array}\right) \times\left(\begin{array}{c}
s_{0}^{r} \\
s_{0}^{i} \\
s_{1}^{r} \\
s_{1}^{i} \\
s_{2}^{r} \\
s_{2}^{i}
\end{array}\right)=\left(\begin{array}{c}
f^{r} \\
f^{i} \\
b^{r} \\
b^{i} \\
e^{r} \\
e^{i}
\end{array}\right)
$$

The residual recurrences are: 


$$
\begin{aligned}
w_{0, r}^{(j)} & =2\left[w_{0, r}^{(j-1)}-d_{0, r}^{(j-1)}+a^{r} d_{1, r}^{(j-1)}-a^{i} d_{1, i}^{(j-1)}+c^{r} d_{2, r}^{(j-1)}-c^{i} d_{2, i}^{(j-1)}\right] \\
w_{0, i}^{(j)} & =2\left[w_{0, i}^{(j-1)}-d_{0, i}^{(j-1)}+a^{i} d_{1, r}^{(j-1)}+a^{r} d_{1, i}^{(j-1)}+c^{i} d_{2, r}^{(j-1)}+c^{r} d_{2, i}^{(j-1)}\right] \\
w_{1, r}^{(j)} & =2\left[w_{1, r}^{(j-1)}-d_{1, r}^{(j-1)}\right] \\
w_{1, i}^{(j)} & =2\left[w_{1, i}^{(j-1)}-d_{1, i}^{(j-1)}\right] \\
w_{2, r}^{(j)} & =2\left[w_{2, r}^{(j-1)}-d_{2, r}^{(j-1)}\right] \\
w_{2, i}^{(j)} & =2\left[w_{2, i}^{(j-1)}-d_{2, i}^{(j-1)}\right]
\end{aligned}
$$

with the initial conditions

$$
w_{0, r}^{(0)}=f^{r}, \quad w_{0, i}^{(0)}=f^{i}, \quad w_{1, r}^{(0)}=b^{r}, \quad w_{1, i}^{(j)}=b^{i}, \quad w_{2, r}^{(j)}=e^{r}, \quad w_{2, i}^{(j)}=e^{i}
$$

The convergence requires that the following conditions are satisfied

$$
\left|a^{r}\right|+\left|a^{i}\right|+\left|c^{r}\right|+\left|c^{i}\right| \leq \alpha,\left|f^{r}\right|,\left|f^{i}\right|, \quad\left|b^{r}\right|,\left|b^{i}\right|,\left|e^{r}\right|,\left|e^{i}\right| \leq 3 / 2
$$

From condition (??) and using $\Delta=1 / 8$, we get $\alpha \leq 3 / 16$ and $\left|a^{r}\right|,\left|a^{i}\right|,\left|c^{r}\right|,\left|c^{i}\right| \leq 3 / 64$ to assure convergence of the algorithm. This range reduction can be achieved by scaling of the initial values.?

A scheme for implementing the $C S P$ operator is shown in Figure ??(a) and the corresponding elementary unit $(E U)$ is illustrated in Figure ??(b). A bit-parallel bus transmits $a$ and $c$, while the real and imaginary parts of $f, b$ and $e$ are loaded in separate cycles. Note that the initialization cycles could be shorter than the iteration cycles.

A block diagram of elementary unit EU0r (real part only) for the CSP operator is shown in Figure ??(b). The modules used are:

- Registers (6)

- Multiple generators MG (4), producing $\{-1,0,1\} \times a^{r}$ and $\{-1,0,1\} \times a^{i}$, and buffers

- Multiplexers MUX (2) for initializing the residual

- A [6:2] adder (the shaded MS part performs the indicated subtraction of the selected digit)

- Output digit selection $S E L$ (a small table or a gate network)

As in the $C M A$ operator, the digit-serial outputs of the $E U 0$ can be converted into digit-parallel form using on-the-fly converters $\mathrm{OFCr}$ and $\mathrm{OFCi}$. The cycle time, in terms of a full adder (complex gate) delay $t$, is estimated as

$$
\begin{aligned}
T_{E U-C S P} & =t_{B U F F}+t_{M G}+t_{S E L}+t_{[6: 2]}+t_{R E G} \\
& \approx(0.4+0.3+1+2.3+0.9) t=4.9 t
\end{aligned}
$$

The cost, again in terms of area of a full adder $A_{F A}$, is estimated as CHANGE

$$
\begin{aligned}
A_{E U-C S P}(m) & =A_{S E L}+4 A_{B U F F}+(m+2)\left[4 A_{M G}\right. \\
& \left.+A_{M U X}+A_{[6: 2]}+6 A_{R E G}+2 A_{O F C}\right] \\
& \approx[5+4 \times 0.4+(m+2)(5 \times 0.45 \\
& +4.3+6 \times 0.6+2 \times 2.1)] A_{F A} \\
& \approx(35+14 m) A_{F A}
\end{aligned}
$$



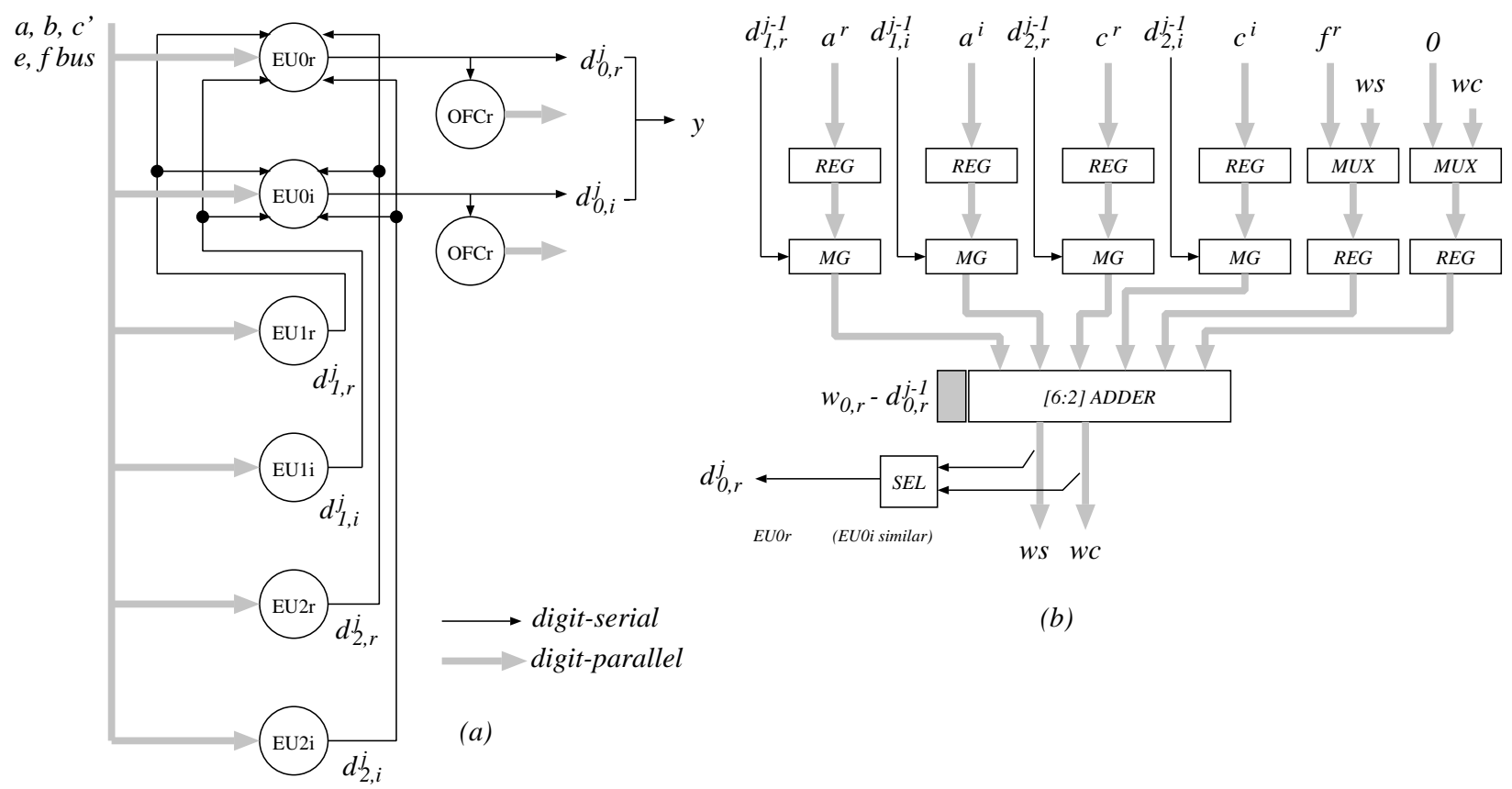

Figure 2. (a) Overall scheme for CSP operator. (b) Block diagram of elementary unit.

As discussed in the previous section, the cost is estimated as the area occupied by the modules using the area of a full-adder $A_{F A}$ as the unit. A total cost of an $m$-bit $C S P$ operator is

$$
A_{C S P}(m)=2 \times A_{E U-C S P}(m)+4 \times(m+2) A_{R E G} \approx(70+30 m) A_{F A}
$$

\section{COMPLEX SUM OF SQUARES OPERATOR (CSS)}

This operator computes $y=a^{2}+b^{2}$. In the real domain, the mapping to a linear system is

$$
\left(\begin{array}{ccc}
1 & -a & -b \\
0 & 1 & 0 \\
0 & 0 & 1
\end{array}\right) \times\left(\begin{array}{l}
s_{0} \\
s_{1} \\
s_{2}
\end{array}\right)=\left(\begin{array}{l}
0 \\
a \\
b
\end{array}\right)
$$

and the solution $s_{0}=y$. In the complex domain, $a=a^{r}+i a^{i}$, and $b=b^{r}+i b^{i}$. The mapping in this case is

$$
\left(\begin{array}{cccccc}
1 & 0 & -a^{r} & a^{i} & -b^{r} & b^{i} \\
0 & 1 & -a^{i} & -a^{r} & -b^{i} & -b^{r} \\
0 & 0 & 1 & 0 & 0 & 0 \\
0 & 0 & 0 & 1 & 0 & 0 \\
0 & 0 & 0 & 0 & 1 & 0 \\
0 & 0 & 0 & 0 & 0 & 1
\end{array}\right) \times\left(\begin{array}{c}
s_{0}^{r} \\
s_{0}^{i} \\
s_{1}^{r} \\
s_{1}^{i} \\
s_{2}^{r} \\
s_{2}^{i}
\end{array}\right)=\left(\begin{array}{c}
0 \\
0 \\
a^{r} \\
a^{i} \\
b^{r} \\
b^{i}
\end{array}\right)
$$

The residual recurrences are: 


$$
\begin{aligned}
& w_{0, r}^{(j)}=2\left[w_{0, r}^{(j-1)}-d_{0, r}^{(j-1)}+a^{r} d_{1, r}^{(j-1)}-a^{i} d_{1, i}^{(j-1)}+b^{r} d_{2, r}^{(j-1)}-b^{i} d_{2, i}^{(j-1)}\right] \\
& w_{0, i}^{(j)}=2\left[w_{0, i}^{(j-1)}-d_{0, i}^{(j-1)}+a^{i} d_{1, r}^{(j-1)}+a^{r} d_{1, i}^{(j-1)}+b^{i} d_{2, r}^{(j-1)}+b^{r} d_{2, i}^{(j-1)}\right] \\
& w_{1, r}^{(j)}=2\left[w_{1, r}^{(j-1)}-d_{1, r}^{(j-1)}\right] \\
& w_{1, i}^{(j)}=2\left[w_{1, i}^{(j-1)}-d_{1, i}^{(j-1)}\right] \\
& w_{2, r}^{(j)}=2\left[w_{2, r}^{(j-1)}-d_{2, r}^{(j-1)}\right] \\
& w_{2, i}^{(j)}=2\left[w_{2, i}^{(j-1)}-d_{2, i}^{(j-1)}\right]
\end{aligned}
$$

with the initial conditions

$$
w_{0, r}^{(0)}=0, \quad w_{0, i}^{(0)}=0, \quad w_{1, r}^{(0)}=a^{r}, \quad w_{1, i}^{(j)}=a^{i}, \quad w_{2, r}^{(j)}=b^{r}, \quad w_{2, i}^{(j)}=b^{i}
$$

The convergence requires that the following conditions are satisfied

$$
\left|a^{r}\right|+\left|a^{i}\right|+\left|b^{r}\right|+\left|b^{i}\right| \leq \alpha
$$

From condition (??) and using $\Delta=1 / 8$, we get $\alpha \leq 3 / 16$ and $\left|a^{r}\right|,\left|a^{i}\right|,\left|b^{r}\right|,\left|b^{i}\right| \leq 3 / 64$ to assure convergence of the algorithm. This range reduction can be achieved by scaling of the initial values.

A scheme for implementing the $C S S$ operator (general and elementary unit) is similar to that of Figure ??. Consequently the delays and the cost are similar as estimated for the CSP operator.

\section{COMPLEX INTEGER POWERS OPERATOR $(C I P)$}

The operator computes in the real domain consecutive integer powers of the argument $x$ in parallel: $x^{2}, x^{3}, \ldots$, $x^{k}$. The corresponding linear system is

$$
\left(\begin{array}{ccccccc}
1 & -x & 0 & 0 & 0 & \cdots & 0 \\
0 & 1 & -x & 0 & 0 & \cdots & 0 \\
\vdots & \vdots & \vdots & \vdots & \vdots & \cdots & \vdots \\
0 & 0 & \cdots & 0 & 0 & 1 & -x \\
0 & 0 & 0 & \cdots & 0 & 0 & 1
\end{array}\right) \times\left(\begin{array}{l}
s_{0} \\
s_{1} \\
\vdots \\
s_{k-1} \\
s_{k}
\end{array}\right)=\left(\begin{array}{c}
0 \\
0 \\
\vdots \\
0 \\
0 \\
x
\end{array}\right)
$$

and the integer powers are obtained as

$$
s_{0}=x^{k}, s_{1}=x^{k-1}, \ldots, s_{n-1}=x^{2}
$$

The mapping in the complex domain is shown next. The complex argument is $z=x+i y$. 


$$
A=\left(\begin{array}{cccccccccc}
1 & 0 & -x & y & 0 & 0 & 0 & 0 & \cdots & 0 \\
0 & 1 & -y & -x & 0 & 0 & 0 & 0 & \cdots & 0 \\
0 & 0 & 1 & 0 & -x & y & 0 & 0 & \cdots & 0 \\
0 & 0 & 0 & 1 & -y & -x & 0 & 0 & \cdots & 0 \\
\vdots & \vdots & \vdots & \vdots & \vdots & \vdots & \vdots & \vdots & \vdots & \vdots \\
0 & 0 & \cdots & 0 & 0 & 0 & 1 & 0 & -x & y \\
0 & 0 & \cdots & 0 & 0 & 0 & 0 & 1 & -y & -x \\
0 & 0 & \cdots & 0 & 0 & 0 & 0 & 0 & 1 & 0 \\
0 & 0 & \cdots & 0 & 0 & 0 & 0 & 0 & 0 & 1
\end{array}\right)
$$

The components of the solution $s$ of the linear system

$$
\begin{gathered}
A \times\left(s_{0}^{r}, s_{0}^{i}, s_{1}^{r}, s_{1}^{i}, \ldots, s_{k-1}^{r}, s_{k-1}^{i}, s_{k}^{r}, s_{k}^{i}\right)^{T} \\
=(0,0,0,0, \ldots, 0,0, x, y)^{T}
\end{gathered}
$$

are equal to the integer powers of $z$.

The residual recurrences are

$$
\begin{aligned}
& w_{h, r}^{(j)}=2\left[w_{h, r}^{(j-1)}-d_{h, r}^{(j-1)}+x d_{h+1, r}^{(j-1)}-y d_{h+1, i}^{(j-1)}\right] \\
& w_{h, i}^{(j)}=2\left[w_{h, i}^{(j-1)}-d_{h, i}^{(j-1)}+y d_{h+1, r}^{(j-1)}+x d_{h+1, i}^{(j-1)}\right]
\end{aligned}
$$

for $h=k$,

$$
\begin{aligned}
& w_{k, r}^{(j)}=2\left[w_{k, r}^{(j-1)}-d_{k, r}^{(j-1)}\right] \\
& w_{k, i}^{(j)}=2\left[w_{k, i}^{(j-1)}-d_{k, i}^{(j-1)}\right]
\end{aligned}
$$

with the initial conditions for $h=0, \ldots, k-1$

$$
w_{h, r}^{(0)}=0, w_{h, i}^{(0)}=0
$$

and

$$
w_{k, r}^{(0)}=x, w_{k, i}^{(0)}=y
$$

The convergence requires that the following conditions are satisfied

$$
|x|+|y| \leq \alpha
$$

From condition (??) and using $\Delta=1 / 8$, we get $\alpha \leq 3 / 16$ and $|x|,|y| \leq 3 / 32$ to assure convergence of the algorithm. This range reduction can be achieved by scaling of the initial values.

A scheme for implementing the CIP operator is shown in Figure ??. The corresponding elementary unit is similar to the $E U$ of the $C M A$ operator, illustrated in Figure ??(b), with the same cycle time and cost. A bit-parallel bus transmits $x$ and $y$ values in a broadcast mode as discussed earlier. A total cost of an $m$-bit $C I P k$ operator is

$$
A_{C I P k}(m)=(k-1) \times A_{E U-C M A}(m)+2 \times(m+2) A_{R E G}
$$

The same scheme, with different initialization, can be used to evaluate complex polynomials. 


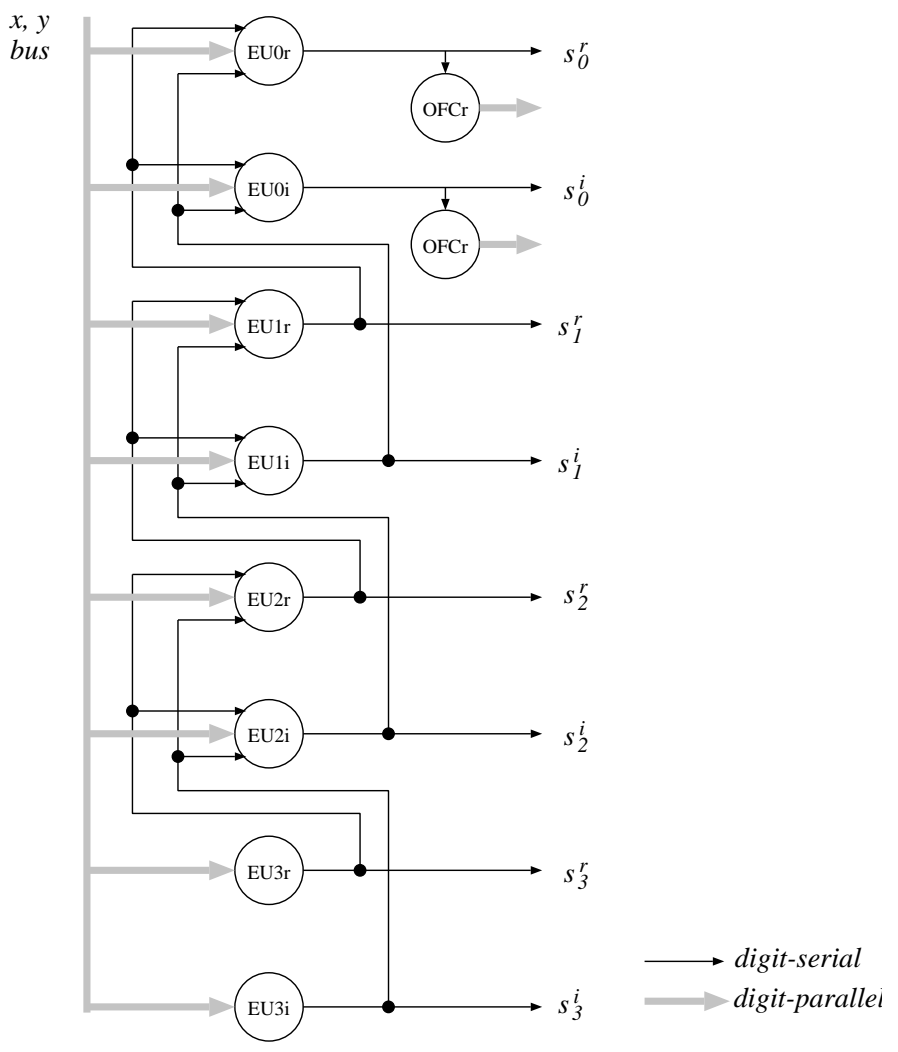

Figure 3. Scheme for $C I P$ operator.

\section{SUMMARY}

We presented a general method for computing several commonly used arithmetic expressions in the complex domain: multiply-add, sum of products, sum of squares, and integer powers. The method consists of mapping the operators on diagonally-dominant systems of complex linear equations, transforming the system from the complex to the real domain, and solving it using digit-by-digit MSDF algorithm. The latency is roughly $m$ cycles for $m$ bits of precision and independent of the order of the resulting linear system. The cycle time is independent of $m$. We discussed the mapping of operators to linear systems, transforms from the real to the complex domains, the recurrences and convergence conditions. Implementations of the proposed operators are discussed at a high level with estimates of the cost and cycle time. The method used here has been applied in the case of complex polynomials and rational functions. ?,?

\section{REFERENCES}

1. T. Aoki, H. Amada, and T. Higuchi. Real/complex reconfigurable arithmetic using redundant complex number systems. Proc. 13th IEEE Symposium on Computer Arithmetic, pp.200-207, 1997.

2. M.D. Ercegovac. A general method for evaluation of functions and computation in a digital computer. PhD thesis, Dept. of Computer Science, University of Illinois, Urbana-Champaign, 1975.

3. M.D. Ercegovac. A General Hardware-oriented Method for Evaluation of Functions and Computations in a Digital Computer. IEEE Trans. Comp., C-26(7):667-680, 1977.

4. M.D. Ercegovac and T. Lang, Digital Arithmetic, Morgan Kaufmann Publishers, San Francisco, 2004.

5. M.D. Ercegovac and J.-M. Muller. Complex Division with Prescaling of Operands. IEEE International Conference on Application-Specific Systems, Architectures and Processors, pp. 293-303, 2003. 
6. M.D. Ercegovac and J.-M. Muller, Design of a complex divider. Proc. SPIE on Advanced Signal Processing Algorithms, Architectures, and Implementations XII, pp. 51-59, 2004.

7. M.D. Ercegovac and J.-M. Muller. Complex Square Root with Operand Prescaling. IEEE International Conference on Application-Specific Systems, Architectures and Processors, pp. 293-303, 2004.

8. M.D. Ercegovac and J.-M. Muller, Solving Systems of Linear Equations in Complex Domain : Complex E-Method. LIP Report No. 2007-2, École Normale Supérieure de Lyon, France.

9. M.D. Ercegovac and J.-M. Muller, A Hardware-Oriented Method for Evaluating Complex Polynomials. IEEE International Conference on Application-Specific Systems, Architectures and Processors, 2007.

10. R.D. McIlhenny, Complex Number On-line Arithmetic for Reconfigurable Hardware: Algorithms, Implementations, and Applications, Ph.D. Dissertation, Computer Science Department, University of California, 2002.

11. V. Oklobdzija, D. Villeger and T. Soulas, An Integrated Multiplier for Complex Numbers. J. of VLSI Signal Processing, vol.7, no. 3, pp.213-222, May 1994.

12. B.W.Y. Wei, H. Du, and H. Chen, A Complex-Number Multiplier Using Radix-4 Digits. Proc. 12th IEEE Symposium on Computer Arithmetic, pp. 84-90, 1995 